\title{
Realization of 2-Dimensional Air-Bridge Silicon Photonic Crystals by Focused Ion Beam, Milling and Nanopolishing
}

\author{
Wico C.L. Hopman ${ }^{\mathrm{a}}{ }^{*}$, René M. de Ridder ${ }^{\mathrm{a}}$, Shankar Selvaraja ${ }^{\mathrm{a}}$, Cazimir G. Bostan ${ }^{\mathrm{a}, \mathrm{c}}$, Vishwas J. \\ Gadgil $^{\mathrm{a}}$, Laurens Kuipers ${ }^{\mathrm{a}, \mathrm{b}}$, Alfred Driessen ${ }^{\mathrm{a}}$ \\ ${ }^{a}$ MESA+ Research Institute, University of Twente, P.O. Box 217, 7500 AE Enschede, The \\ Netherlands; \\ ${ }^{\mathrm{b}}$ FOM Institute for Atomic and Molecular Physics (AMOLF), Kruislaan 407, 1098 SJ Amsterdam, \\ The Netherlands; \\ ${ }^{\mathrm{c}}$ Now at Honeywell Romania SRL, 15 Oltetului Street, 023817-Bucharest, Romania
}

\begin{abstract}
We report the design and fabrication of small photonic crystal structures which are combined with conventional dielectric ridge waveguides. We describe in details the fabrication of both rough and smooth membranes, which are used as host for photonic crystals. Two Focused Ion Beam milling experiments are highlighted: the first one shows how photonic crystals can be fast and accurate milled into a Si membrane, whereas the second experiment demonstrates how focused ion beam milling can turn a rough surface into a well-patterned nano-smooth surface. The previously ultra rough surface showed no detectable roughness after milling due to the nanopolishing effect of the focused ion beam milling.
\end{abstract}

Keywords: Photonic crystals, Focused Ion Beam, Nanopolishing, Hexagonal Holes, SOI, Membranes

\section{INTRODUCTION}

Photonic crystals are structures with a periodic dielectric function, which may result in the prohibition of the propagation of electromagnetic waves for certain frequencies in all crystal directions: the photonic bandgap. In this paper we investigate a 2-dimensional triangular pattern of hexagonal holes in a high index silicon membrane. When introducing 'defects' in photonic crystal slabs (PCS) in a controlled manner, this arrangement becomes suitable for design and implantation of passive and active building blocks (e.g. filters ${ }^{1}$, sensors ${ }^{2}$, lasers ${ }^{3}$ ). The size of the bandgap of the uniform crystal area not only depends on the refractive index contrast but also on the air filling factor of the photonic crystal, which is determined by the size of the holes compared to the lattice constant $(a)$. It is well known from the literature $^{4}$ that a complete bandgap, i.e. a stopband for all polarizations and directions, can be maximized by creating a crystal having small islands of high-index dielectric, which are interconnected through narrow veins. Previously we calculated $^{5}$ that using hexagonal holes rotated by 9 degrees with respect to the lattice can result in an optimized complete bandgap. This means that for a typical design with lattice parameter $\sim 500 \mathrm{~nm}$ the width of the veins (i.e. the thickness of the sidewalls separating adjacent holes), can be as small as $\sim 50 \mathrm{~nm}$ or even less. This calls for fabrication with nanometer precision, which is not easily achieved with most lithographic techniques. Using a focused ion beam milling machine (dual beam Nova 600) with a spot size of $\sim 12 \mathrm{~nm}$ and an address grid resolution of $3.1 \mathrm{~nm}$ we were able to fabricate these structures.

Focused ion beam (FIB) processing is a well-established technique with the unique capabilities to locally sputter etch, ion implant, and deposit metals and insulators with a feature size in the order of nanometers, without the need of a mask $^{6}$. In optoelectronics, FIB has been applied for defining the end facet mirrors of conventional semiconductor lasers ${ }^{7}$, ${ }^{8}$. It also has been applied for bulk micromachining of macro-porous silicon in order to fabricate 3D Yablonovite-like photonic crystals ${ }^{9}$, and recently for fabricating $2 \mathrm{D}$ periodic structures in III-V compounds ${ }^{10}$. Another recent application

\footnotetext{
W.C.L.Hopman@ewi.utwente.nl; phone 0031-53-489-4440; fax 0031-53-4893343; http://ioms.ewi.utwente.nl/
} 
of FIB has been the fabrication of quasi-1D photonic crystals by milling 1D gratings into freestanding multilayer membranes $^{11}$. An advantage of FIB milling, compared to direct e-beam writing and deep-UV lithography, is that it does not need proximity correction if used on conducting surfaces. Moreover, it provides direct definition of a pattern either directly into the target layer or into an etch resistant mask, and it can be integrated with optical lithography. Finally, similar to its use with electronic circuits, FIB can be used for repairing or trimming optical waveguides and resonator structures.

For photonic (crystal) structures which require the definition of sub-micron features in high-index contrast material systems, a particularly attractive property of FIB is the nanopolishing effect that we observed, resulting in smooth structures which are hard to obtain with other etching methods (e.g. reactive ion etching - RIE). The nanopolishing effect is a complex process of ion-implantation, amorphization, and re-deposition of nano-crystalline silicon ${ }^{12}$. Although promising photonic crystal structures have been FIB-fabricated in thin slabs, and especially in free-standing membranes, it is still a challenge for FIB to obtain deep holes with extremely high aspect ratios. Other issues important for photonic devices are charge-related proximity effects on insulating substrates, amorphization and implantation of $\mathrm{Ga}+$ ions ${ }^{12,13}$ which deserves attention by optical investigation. It should be noted that these FIB induced effects can be minimized by merely using the FIB as lithography tool, as is outlined by figure 1.

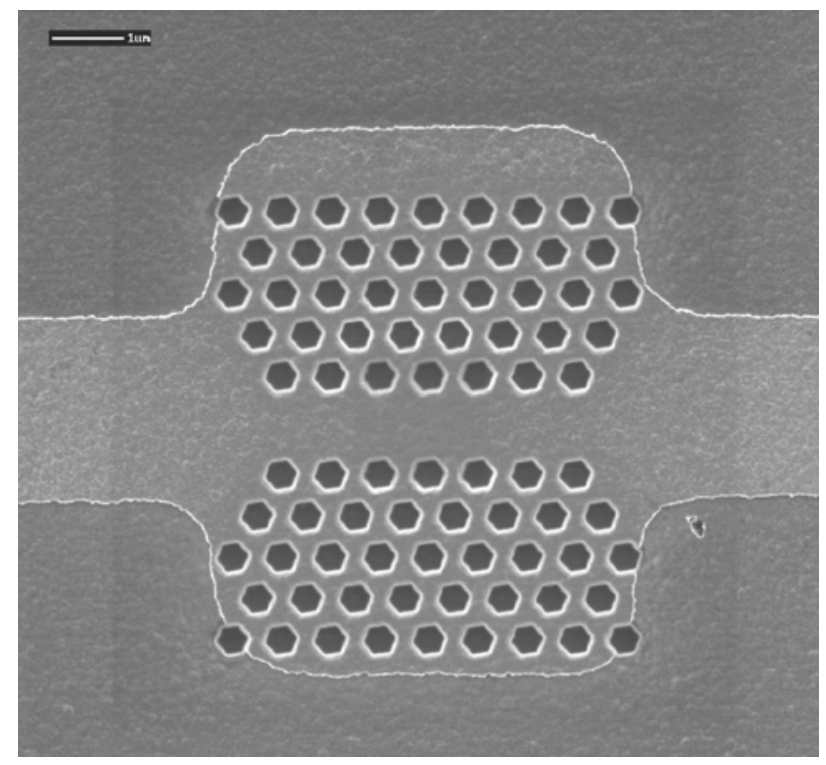

Figure 1. FIB-milled hexagon-type photonic crystal chromium pattern on silicon using a Nova 200 single beam FIB (FEI Company).

FIB machines can resolve a limited number of "pixels", so that a compromise must be found between resolution and the accessible area for processing (field-of-view, FoV). The processed area can always be extended by mechanical specimen translation, but this requires additional alignment procedures and may introduce stitching errors. There are several photonic structures of interest that fit entirely within the high-resolution FoV of a few tens of microns square. For larger structures the most promising application of FIB is the combination with large-area lithographic techniques e.g. mask-based deep-UV, where sub-structures may be FIB-defined. This type of fast prototyping allows for testing of many variations on a prefabricated generic photonic crystal "breadboard", avoiding the typical long turn-around times for mask-based lithography. It can also be used for trimming/tuning existing structures, e.g. resonators and their coupling coefficients, and critical waveguide junctions/facets. In this paper we will first present the design and fabrication scheme of the host structures: the crystalline silicon membranes from silicon-on-insulator (SOI) wafers. Furthermore we will discuss two fabrication methods for removing the $\mathrm{SiO}_{2}$ that result in either smooth or extremely rough surfaces in section 2: Fabrication. Also a detailed description of the important parameters for the milling experiments will be given. The fabrication result of a photonic crystal lattice milled into the membrane is presented in section 3: FIB milling results. A demonstration of the FIB induced nanopolishing effect will be also be given. Finally we will present the main conclusions. 


\section{FABRICATION}

In this section we will first briefly describe the design and fabrication of the photonic host structures needed for the FIB milling process. Secondly we will describe the FIB milling process and its relevant parameters.

\subsection{Host structure definition}

As a host structure for FIB milling we focused on symmetric air-bridge type PCS ${ }^{14}$ in silicon (Si) membranes that are fabricated from commercially available SOI wafers ${ }^{15}$ with a Si device top-layer etched back to $\sim 300 \mathrm{~nm}$ on top of a $3.3 \mu \mathrm{m} \mathrm{SiO} 2$ layer. The fabrication of a membrane starts with (UV) optical lithography for defining the etch windows, and the alignment markers. Next RIE is used to etch the top Si layer. The PCS area (host-area) and the waveguides are defined by a second lithography step. Another dry-etching step is used to etch the pattern into the silicon, see figure 2.

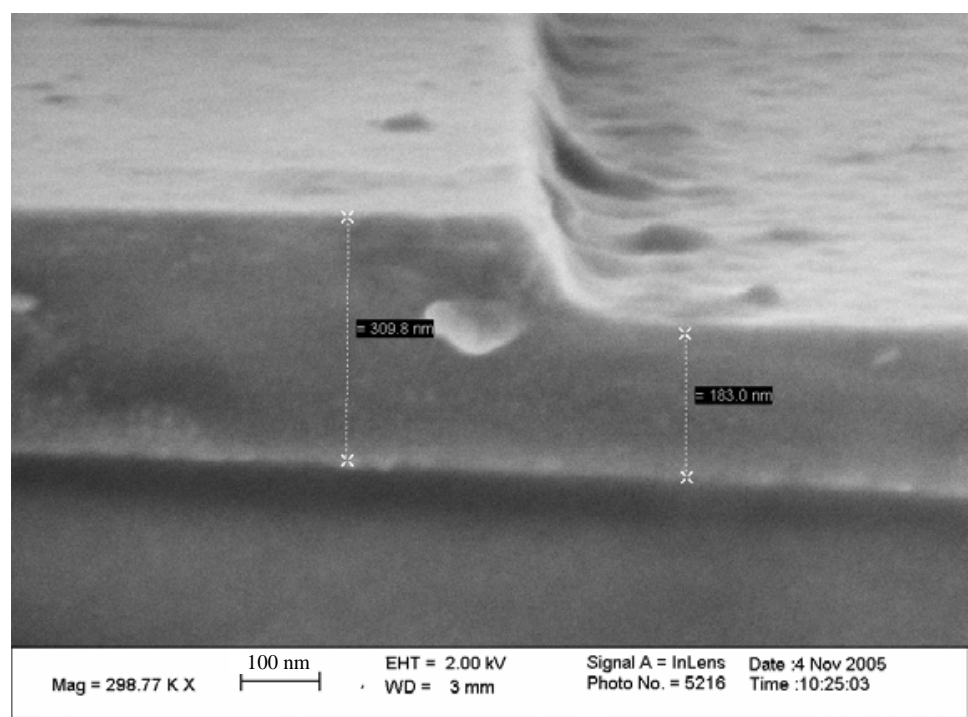

Figure 2. A cross-section of the fabricated ridge waveguide in SOI, showing relatively large sidewall roughness. This particular waveguide has a ridge of $\sim 120 \mathrm{~nm}$.

We have chosen to use low-lateral-contrast access waveguides which can be single-moded at a width larger than $1 \mu \mathrm{m}$, thus avoiding the need for expensive e-beam generated masks. Therefore, fabrication of our design called for a relatively slow RIE process in order to produce the needed shallow ( $20 \mathrm{~nm})$ ridge with sufficient accuracy. However, our laser-beam generated mask provided poor edge definition, which induced large side-wall roughness, although the overall loss parameter of the waveguides is still low, because the side-wall area is very small compared to the case of photonic wires. To obtain both smooth and highly roughened (porous ${ }^{16}$ ) surfaces we have fabricated the membranes in two different ways. The former is obtained by applying a third lithography step to protect the top silicon during the buffered $\mathrm{HF}$ etch of the bottom sacrificial $\mathrm{SiO}_{2}$ layer in a dark environment to form the final membranes. The roughened sample is fabricated by BHF etch without protecting layer and by exposure of light to porosify the Si surface of the membranes (see figure 5 - 7). The sample shows pores (roughness) with an average around 100 nm. For integrated optics such a roughness is clearly unwanted. In this research we will show that after processing, the target area becomes smooth with an average roughness of less than $5 \mathrm{~nm}$.

\subsection{FIB milling of Si membranes}

A recently introduced dual-beam FIB machine, the dual beam FEI Nova 600, was used for the FIB processing of our structures. It has both an ion and e-beam column, which can be used simultaneously, e.g. for charge neutralization, or separately for milling followed by a destruction free inspection using the e-beam. For the experiments presented in this paper the magnification was set to $10000(10 \mathrm{kX})$, which gives a pixel allocation size of $3.1 \mathrm{~nm}$. The total field that 
could be written without stitching errors (FoV) was about $12.7 \mu \mathrm{m}$ x $12.7 \mu \mathrm{m}$. The acceleration voltage was fixed as well as the dwell time (time that the beam is stationary at a certain pixel) to respectively $30 \mathrm{kV}$ and $100 \mu \mathrm{s}$. For definition of sub-micron structures it is important to use low milling currents to minimize charging and re-deposition effects; also a lower current gives a smaller 1/e spot size. The downside to a small etch current is the total etch time becomes longer and consequently the milling process will be more sensitive for distortion by drift. In our experiment the etch times were typically about 5 minutes per prototype. We used two different ion-beam current values which provide a good compromise between milling speed and accuracy, viz. 28 pA resulting in a spot size (1/e beam diameter) of $~ 15.5$ $\mathrm{nm}$, and $9.7 \mathrm{pA}$ which gives a spot size of $11.9 \mathrm{~nm}$. For nano-structures the (pixel) sequence of milling may become important, i.e. the temporarily local geometry affects the final result. In this investigation we have fixed the milling direction of the ion beam by writing the design in horizontal lines, thus avoiding an additional degree of complexity.

\section{FIB MILLING RESULTS}

In section 3.1 we present a PCS that has been designed for characterization of the complete bandgap of rotated hexagonal holes in a triangular lattice. The milling experiment, performed at $28 \mathrm{pA}$, shows that the FIB is a valuable tool for easy and fast definition of integrated photonic functions, especially suited for photonic crystals in high-index contrast systems, since they only require small writing areas. Another interesting phenomenon, the observed nanopolishing effect is discussed in section 3.2. We will show a clear example for this nanopolishing effect.

\subsection{Planer photonic crystal for optical characterization}

For optical transmission experiments we have fabricated a lateral multimodal waveguide of $\sim 10 \mu \mathrm{m}$ width having a thicker region $(\sim 12 \mu \mathrm{m})$ exactly in the middle of the membrane. The ridge waveguide gives a lateral mode spread (about 6 modes) over a small index difference, which is preferred for accurate detection of the band edges.

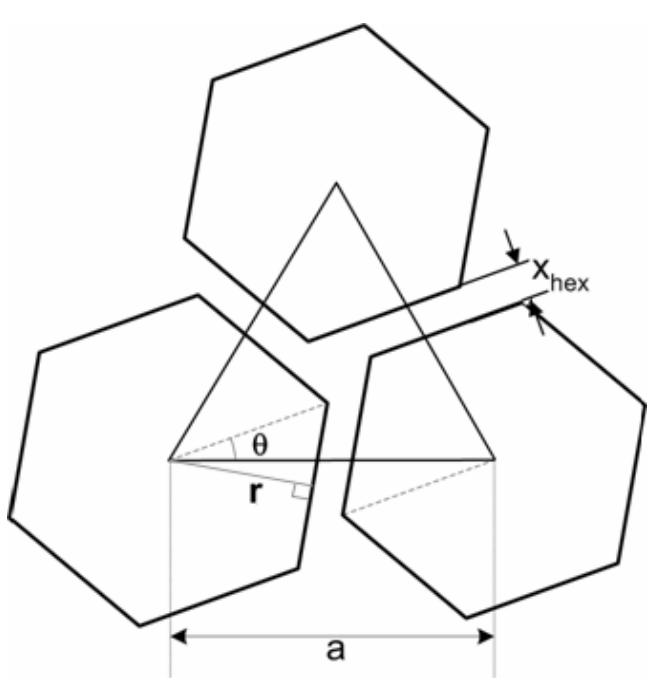

Figure 3. Schematic drawing of the triangular lattice with hexagonal holes rotated over an angle $\theta$.

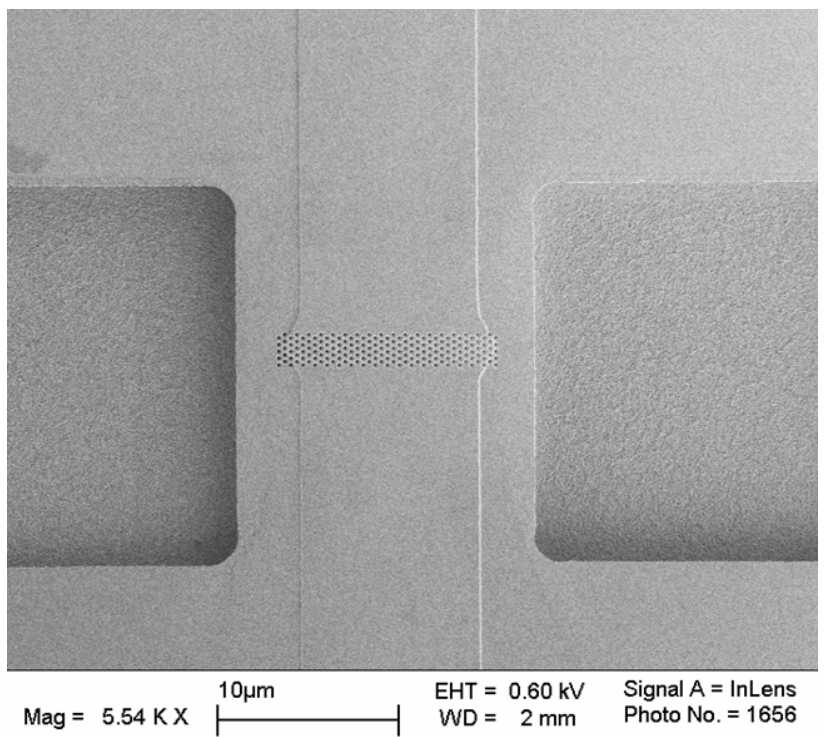

Figure 4. Top view of a 5-periods photonic crystal structure, designed for transmission measurements

The lattice constant $a$ used for this design was $380 \mathrm{~nm}$ and the radius (r) was designed as $r / a=0.25$ using the definition of the unit cell as shown in figure 3. A top view image of the milled planar photonic crystal (PhC) region plus waveguide section and etch windows (used for bottom oxide removal) is shown in figure 4. A SEM photo with higher magnifications of the PhC area is shown in figure 5 and 6. 


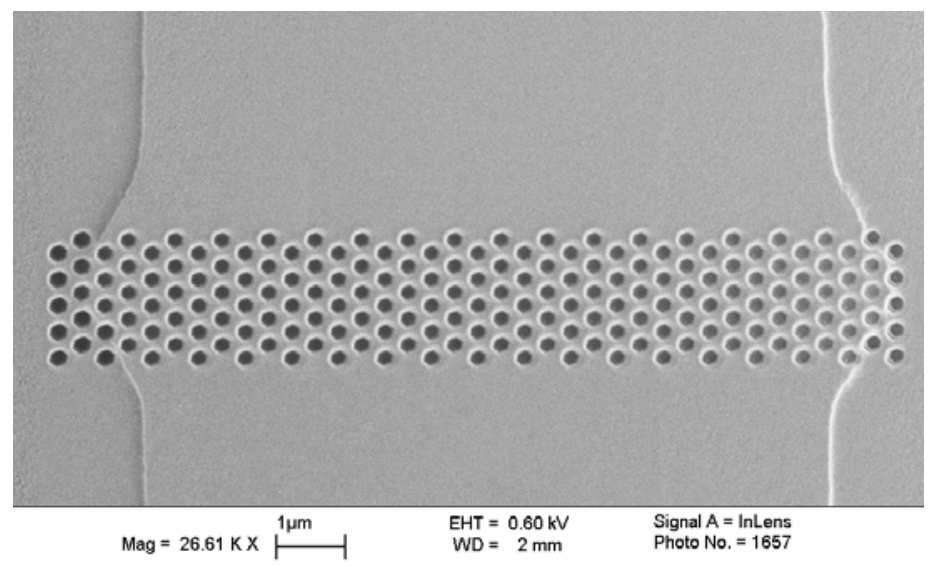

Figure 5. A zoomed in top view of the photonic crystal section shown figure 4.

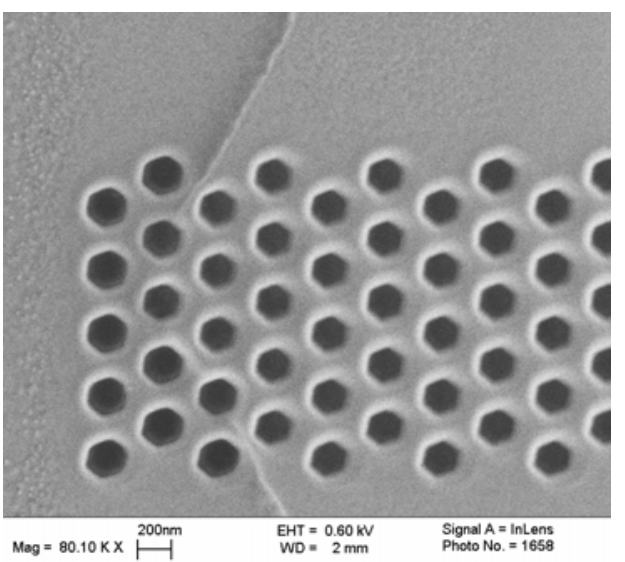

Figure 6. A (further zoom in) top view of the photonic crystal section shown in the left graph (figure 5)

A 5-periods long and 37 “layers” wide $\Gamma$-K oriented crystal was chosen. After analyzing the SEM photo's, we find a value of $380 \mathrm{~nm} \pm 10 \mathrm{~nm}$ for $a$, and an average $r / a$ of the holes of r/a $\sim 0.24 \pm 0.2$. The fabricated structures match well with the design. However some variations are found in hole size in the region outside the ridge waveguide, see figure 6. This is caused by the smaller silicon thickness outside the ridge region which requires a smaller milling time. Since changing the current locally is not an option (loose of focus), a possible solution is to vary either the dwell times of the individual holes or simply modify the total number of pixels representing the hole area. The latter approach may lead to a decrease of the definition accuracy. The $28 \mathrm{pA}$ milling current used for this design (spot size $15.5 \mathrm{~nm}$ ) causes some rounding of the hexagons, due to beam overlap. A further decrease of the beam current to 9.7 pA will sharpen the hexagons, but the milling time will also triple to $\sim 15$ minutes.

\subsection{Nanopolishing effect}

In section 2.1 we have described the method to achieve roughened membranes. The result is presented in left part of figure 7, which shows the porosification of the top silicon layer. For clearness we have chosen to use a large air-filling fraction and a larger lattice constant, respectively $r / a \sim 0.4, a=950 \mathrm{~nm}$. Using graphical analysis: edge detection and a Fourier technique on the porous area of the SEM graph, we observe an average pore size of about $100 \mathrm{~nm}$. After etching for $~ 2.5$ minutes at a low current of $9.7 \mathrm{pA}$ we obtain a PhC structure which has not yet been etched through the membrane. Interesting is that the previously rough area has been transformed into a smooth (patterned) surface.

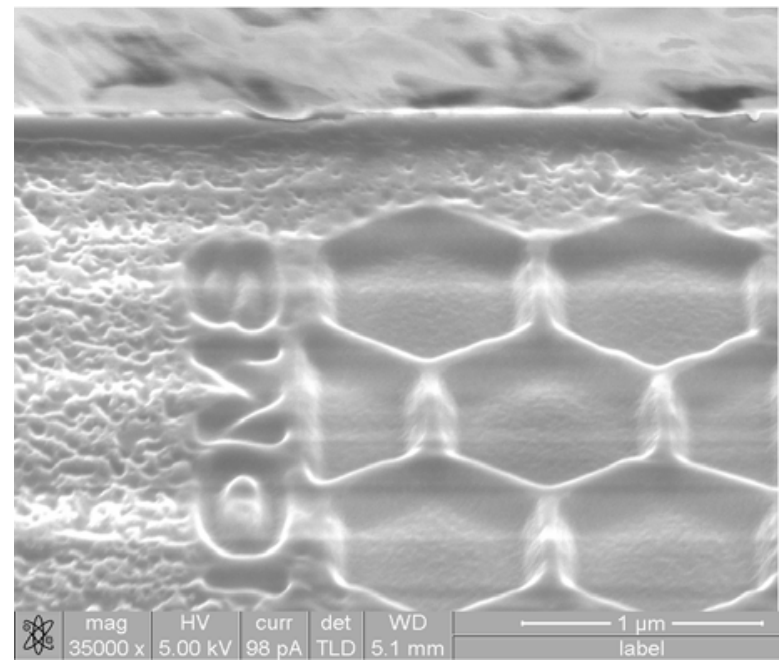

Figure 7. A milled hexagonal structure which shows the nanopolishing effect in and around the holes. 
In fact, the roughness inside the hole has dropped below the accuracy of the FIB-SEM. An AFM measurement method could be used to more exactly quantify the roughness. An interesting idea would to combine both the rough surface (having an extremely large surface) with a photonic crystal structure for sensing purposes. The actual implementation is still to be designed.

We anticipate that the observed nanopolishing is an important property of the FIB, which can be exploited for the realization of nano-smooth photonic crystals. We will report on the impact of FIB-related effects like smoothening, amorphization, and implantation, on the optical properties of a PCS in a different paper.

\section{CONCLUSIONS}

We have shown successful fabrication of low-ridge SOI waveguide structures. The air-bridge structure could be obtained either by protective etching of the $\mathrm{SiO}_{2}$ bugger layer in a dark environment to achieve smooth surfaces or they could be obtained by etching in an unprotected environment, which gives ultra-rough surfaces. The smooth membranes could be used to host photonic crystal prototype structures, in our case a uniform lattice. On the other hand, the membranes having a porous layer could be used to prove the nanopolishing capabilities of the FIB in photonic structures. The hybrid structure having a porous region and a nano-smooth PhC region may also be useful in optical sensing applications. Though the nanopolishing effect has not been optically characterized at the moment, we are convinced of its important role in nanofabrication of high index-contrast photonic devices, where extremely low roughness is required.

\section{ACKNOWLEDGMENTS}

We thank Bert Otter, Eddy Ruiter and Mark Smithers for taking the SEM pictures. Further we would like to thank Frans Segerink for his technical advice on FIB milling. This research is supported by NanoNed, a national nanotechnology program coordinated by the Dutch ministry of Economic Affairs. This research was also supported by the Dutch Technology Foundation STW and the MESA+ Scientific Research Orientation on “Advanced Photonic Structures”.

\section{REFERENCES}

1. P. R. Villeneuve, S. Fan, and J. Joannopoulos, "Microcavities in photonic crystals: Mode symmetry, tunability, and coupling efficiency," Physical Review B, vol. 54, pp. 7837-7842, 1996.

2. W. C. L. Hopman, P. Pottier, D. Yudistira, J. van Lith, P. V. Lambeck, R. M. De La Rue, A. Driessen, H. Hoekstra, and R. M. de Ridder, "Quasi-one-dimensional photonic crystal as a compact building-block for refractometric optical sensors," IEEE Journal Of Selected Topics In Quantum Electronics, vol. 11, pp. 11-16, 2005.

3. S. Noda, M. Yokoyama, M. Imada, A. Chutinan, and M. Mochizuki, "Polarization mode control of twodimensional photonic crystal laser by unit cell structure design," Science, vol. 293, pp. 1123-1125, 2001.

4. J. D. Joannopoulos, R. D. Meade, and J. N. Winn, Photonic crystals: Molding the flow of light, Princeton University Press, 1995.

5. C. G. Bostan and R. M. de Ridder, "Design of photonic crystal slab structures with absolute gaps in guided modes," Journal of Optoelectronics and Advanced Materials, vol. 4, pp. 921-928, 2002.

6. S. Reyntjens and R. Puers, "A review of focused ion beam applications in microsystem technology," Journal of Micromechanics and Microengineering, vol. 11, pp. 287-300, 2001.

7. Q. Ren, B. Zhang, J. Xu, Z. S. Zhang, Y. B. Jin, Y. Qian, and D. P. Yu, "Etched facet and semiconductor/air DBR facet of a AlGaInP laser diode prepared by focused ion beam milling," Solid State Communications, vol. 130, pp. 433-436, 2004. 
8. T. Ito, H. Ishikawa, T. Egawa, T. Jimbo, and M. Umeno, "Fabrication of flat end mirror etched by focused ion beam for GaN-based blue-green laser diode," Japanese Journal of Applied Physics Part 1, vol. 36, pp. 77107711, 1997.

9. A. Chelnokov, K. Wang, S. Rowson, P. Garoche, and J. M. Lourtioz, "Near-infrared Yablonovite-like photonic crystals by focused-ion-beam etching of macroporous silicon," Applied Physics Letters, vol. 77, pp. 29432945, 2000.

10. M. J. Cryan, M. Hill, D. C. Sanz, P. S. Ivanov, P. J. Heard, L. Tian, S. Y. Yu, and J. M. Rorison, "Focused ion beam-based fabrication of nanostructured photonic devices," IEEE Journal Of Selected Topics In Quantum Electronics, vol. 11, pp. 1266-1277, 2005.

11. Y. Fu, N. K. A. Bryan, O. N. Shing, and H. N. P. Wyan, "Influence analysis of dwell time on focused ion beam micromachining in silicon," Sensors and Actuators, A: Physical, vol. 79, pp. 230, 2000.

12. T. L. Matteson, S. W. Schwarz, E. C. Houge, B. W. Kempshall, and L. A. Giannuzzi, "Electron backscattering diffraction investigation of focused ion beam surfaces," Journal of Electronic Materials, vol. 31, pp. 33-39, 2002.

13. B. I. Prenitzer, C. A. Urbanik-Shannon, L. A. Giannuzzi, S. R. Brown, R. B. Irwin, T. L. Shofner, and F. A. Stevie, "The correlation between ion beam/material interactions and practical FIB specimen preparation," Microscopy and Microanalysis, vol. 9, pp. 216-236, 2003.

14. M. Loncar, T. Doll, J. Vuckovic, and A. Scherer, "Design and fabrication of silicon photonic crystal optical waveguides," Journal of Lightwave Technology, vol. 18, pp. 1402-1411, 2000.

15. S.O.I.TEC, "Silicon on Insulator technologies."

16. V. Lehmann and U. Gosele, "Porous Silicon Formation - a Quantum Wire Effect," Applied Physics Letters, vol. 58, pp. 856-858, 1991. 